\title{
A characterization of simplicial spaces by an extension property \\ by
}

Jakub RONDOŠ and Jiří SPURNÝ

Presented by Piotr BILER

Summary. Let $\mathcal{H}$ be a function space on a compact Hausdorff space $K$. We provide a characterization of the simpliciality of $\mathcal{H}$ via an extension property.

1. Introduction. Let $X$ be a compact convex set in a real locally convex space. We write $\mathfrak{A}^{c}(X)$ for the space of all real continuous affine functions on $X$ endowed with the supremum norm. The symbol $\mathfrak{A}^{c}(X)^{+}$stands for the set of nonnegative elements of $\mathfrak{A}^{c}(X)$. In [4], a compact subset of $X$ is called hyper-extremal if it is a union of compact faces (the class of hyper-extremal subsets of $X$ in fact coincides with compact extremal subsets of $X$; see [4, p. 396] or [5, Proposition 2.69]). The set $X$ is said to have the property $(H)$ if

(1.1) there exists a constant $C \in \mathbb{R}$ such that for each hyper-extremal set $D \subseteq X$ and $a \in \mathfrak{A}^{c}(\overline{\mathrm{co}}(D))^{+}$there exists $b \in \mathfrak{A}^{c}(X)^{+}$such that $b=a$ on $D$ and $\|b\|_{K} \leq C\|a\|_{D}$.

It was shown by Batty [4] that $X$ has the property $(H)$ if and only if $X$ is a Choquet simplex. A similar characterization was proven before by Andersen [2], but it was restricted to metrizable compact convex sets. In the present paper we provide a similar characterization for abstractly defined affine functions.

First we collect several definitions and basic facts of Choquet theory on function spaces. For details we refer the reader to [5]. In what follows, let

2020 Mathematics Subject Classification: Primary 46A55.

Key words and phrases: simplicial space, extension property.

Received 24 October 2019; revised 5 December 2019.

Published online 10 January 2020. 
$K$ be a compact Hausdorff space and $\mathcal{H}$ be a function space on $K$, that is, a linear subspace of the space of continuous functions on $K$, endowed with the supremum norm, containing constant functions and separating the points of $K$.

For $x \in K$ we denote by $\mathcal{M}_{x}(\mathcal{H})$ the set of all $\mathcal{H}$-representing measures for $x$, that is,

$$
\mathcal{M}_{x}(\mathcal{H})=\left\{\mu \in \mathcal{M}^{1}(K): f(x)=\int_{K} f \mathrm{~d} \mu \text { for any } f \in \mathcal{H}\right\} .
$$

(Here $\mathcal{M}^{1}(K)$ stands for the set of all Radon probability measures on $K$.) The Choquet boundary $\mathrm{Ch}_{\mathcal{H}}(K)$ of $\mathcal{H}$ is the set

$$
\mathrm{Ch}_{\mathcal{H}}(K)=\left\{x \in K: \mathcal{M}_{x}(\mathcal{H})=\left\{\varepsilon_{x}\right\}\right\},
$$

where $\varepsilon_{x}$ stands for the Dirac measure at a point $x \in K$.

A function $f$ on $K$ satisfying

$$
f(x) \leq \int_{K} f \mathrm{~d} \mu, \quad x \in K, \mu \in \mathcal{M}_{x}(\mathcal{H}),
$$

is termed $\mathcal{H}$-convex. A function $f$ on $K$ is $\mathcal{H}$-concave if $-f$ is $\mathcal{H}$-convex. If $f$ is both $\mathcal{H}$-convex and $\mathcal{H}$-concave, then $f$ is called $\mathcal{H}$-affine. The family of all continuous $\mathcal{H}$-affine functions is denoted by $\mathcal{A}^{c}(\mathcal{H})$. Then $\mathcal{A}^{c}(\mathcal{H})$ is again a function space. Moreover, it is closed and contains $\overline{\mathcal{H}}$.

We recall that given a pair of measures $\mu, \nu \in \mathcal{M}^{1}(K)$, we say that $\mu \prec \mathcal{H} \nu$ if $\mu(f) \leq \nu(f)$ for any $\mathcal{H}$-convex continuous function $f$ on $K$ (see [5. Definition 3.19]). A measure which is $\prec \mathcal{H}$-maximal is called $\mathcal{H}$-maximal.

If $K=X$ is a compact convex set in a locally convex space and $\mathcal{H}=$ $\mathfrak{A}^{c}(X)$, then $\mathcal{H}$ is a function space with $\mathcal{A}^{c}(\mathcal{H})=\mathcal{H}$ and $\operatorname{Ch}_{\mathcal{H}}(X)=\operatorname{ext} X$, the set of all extreme points of $X$ (see [5, Theorem 2.40]).

Let $A$ be a Borel subset of $K$. Then $A$ is called measure convex if $x \in A$ whenever $x \in K$ and $\mu \in \mathcal{M}_{x}(\mathcal{H})$ with $\mu(K \backslash A)=0$. If $A$ is moreover closed, then it is called $\mathcal{H}$-convex. For a subset $F$ of $K$, let

$$
\overline{\mathrm{co}}^{\mathcal{H}}(F)=\bigcap\{C \subseteq K: C \supseteq F, C \text { is } \mathcal{H} \text {-convex }\} .
$$

The subset $F$ is $\mathcal{H}$-convex if and only if $F=\overline{\mathrm{co}}^{\mathcal{H}}(F)$.

A Borel set $A \subseteq K$ is called measure extremal if for any $x \in A$ and any measure $\mu \in \mathcal{M}_{x}(\mathcal{H}), \mu$ is supported by $A$. Closed measure extremal sets are called $\mathcal{H}$-extremal. Finally, we say that $A$ is a Choquet set if it is both measure convex and measure extremal.

The upper envelope of a bounded function $f$ on $K$ is defined as

$$
f^{*}(x)=\inf \{s(x): s \text { is continuous and } \mathcal{H} \text {-concave, } s \geq f\}, \quad x \in K .
$$

It is always an upper semicontinuous $\mathcal{H}$-concave function on $K$, and it coincides with $f$ on $\mathrm{Ch}_{\mathcal{H}}(K)$ for $f$ upper semicontinuous. 
The state space $\mathbf{S}(\mathcal{H})$ of $\mathcal{H}$ is defined as

$$
\mathbf{S}(\mathcal{H})=\left\{\varphi \in \mathcal{H}^{*}: \varphi \geq 0, \varphi(1)=1\right\} .
$$

It is a $w^{*}$-compact convex subset of $\mathcal{H}^{*}$. The function space $\mathcal{H}$ is called simplicial if $\mathbf{S}\left(\mathcal{A}^{c}(\mathcal{H})\right.$ ) is a Choquet simplex (see [5, Theorem 6.54]). As a canonical example of a simplicial function space serves the space $\mathfrak{A}^{c}(X)$ on a simplex $X$ (see [5, Theorem 6.54]). A less obvious example is the following. Let $U \subset \mathbb{R}^{d}$ be an open bounded set. Then the space of all functions continuous on $\bar{U}$ that are harmonic on $U$ is an example of a simplicial function space (see [5, Theorem 13.35]).

The evaluation mapping $\phi$ from $K$ into $\mathbf{S}(\mathcal{H})$ is defined as

$$
\phi: x \mapsto \phi_{x}, \quad x \in K,
$$

where $\phi_{x}$ maps a function $h \in \mathcal{H}$ to the real number $h(x)$. We further define a mapping $\Phi: \mathcal{H} \rightarrow \mathfrak{A}^{c}(\mathbf{S}(\mathcal{H}))$ for $h \in \mathcal{H}$ as

$$
\Phi(h): s \mapsto s(h), \quad s \in \mathbf{S}(\mathcal{H}) .
$$

We point out several important properties of the mappings $\phi$ and $\Phi$. For the proofs of these facts see e.g. [5, Proposition 4.26, Lemma 8.10 and Proposition 8.22].

The mapping $\phi$ is a homeomorphism of $K$ into $\mathbf{S}(\mathcal{H}), \mathbf{S}(\mathcal{H})=\overline{\mathrm{co}}(\phi(K))$ and $\phi\left(\mathrm{Ch}_{\mathcal{H}}(K)\right)=\operatorname{ext} \mathbf{S}(\mathcal{H})$. If $H \subseteq \mathbf{S}(\mathcal{H})$ is $\mathfrak{A}^{c}(\mathbf{S}(\mathcal{H}))$-measure extremal, then $\phi^{-1}(H \cap \phi(K))$ is measure extremal in $K$. Moreover, for each set $F \subseteq K$ we have

$$
\overline{\mathrm{co}}^{\mathcal{H}}(F)=\phi^{-1}(\overline{\mathrm{co}}(\phi(F)) \cap \phi(K)) .
$$

The mapping $\Phi$ is positive, linear and norm-preserving. It is surjective if and only if $\mathcal{H}$ is closed, and in this case the inverse mapping is realized by

$$
\Phi^{-1}(F)=F \circ \phi, \quad F \in \mathfrak{A}^{c}(\mathbf{S}(\mathcal{H})) .
$$

If $F$ is a closed subset of $K$ then the space $\left.\mathcal{H}\right|_{F}$ of all restrictions of functions from $\mathcal{H}$ to the set $F$ is again a function space.

2. Characterization of simpliciality. We note that from the definition of $\mathcal{H}$-affine functions it follows that the sets of representing measures are the same with respect to both function spaces $\mathcal{H}$ and $\mathcal{A}^{c}(\mathcal{H})$, that is, for each $x \in K$ we have $\mathcal{M}_{x}(\mathcal{H})=\mathcal{M}_{x}\left(\mathcal{A}^{c}(\mathcal{H})\right)$. From this it follows that $\mathcal{A}^{c}\left(\mathcal{A}^{c}(\mathcal{H})\right)=\mathcal{A}^{c}(\mathcal{H})$, and also that the classes of measure extremal and measure convex sets are the same with respect to both these function spaces.

We say that the function space $\mathcal{H}$ has the property $(H)$ if

(2.1) there exists a constant $C \geq 0$ such that for each $\mathcal{H}$-extremal set $D \subseteq K$ and $a \in \mathcal{A}^{c}\left(\left.\mathcal{H}\right|_{\overline{\mathrm{CO}}} \mathcal{H}(D)\right)^{+}$there exists $b \in \mathcal{A}^{c}(\mathcal{H})^{+}$with $\left.b\right|_{D}=a$ and $\|b\|_{K} \leq C\|a\|_{D}$. 
In the rest of the paper we show that this property $(H)$ characterizes the concept of simpliciality of a function space.

First we show the connection between our definition of property $(H)$ and the property $(H)$ of a compact convex set as defined by Batty.

Proposition 2.1. If a function space $\mathcal{H}$ has the property $(H)$ in the sense of (2.1) then $\mathbf{S}\left(\mathcal{A}^{c}(\mathcal{H})\right)$ has the property $(H)$ in the sense of (1.1).

Proof. Let $X=\mathbf{S}\left(\mathcal{A}^{c}(\mathcal{H})\right)$. Let $D \subseteq X$ be a nonempty hyper-extremal set and $a \in \mathfrak{A}^{c}(\overline{\mathrm{co}} D)^{+}$. Then $D$ is $\mathfrak{A}^{c}(X)$-extremal (see [5, Proposition 2.69]). We consider the closed function space $\mathcal{A}^{c}(\mathcal{H})$ and the above-defined mappings $\phi: K \rightarrow \mathbf{S}(X)$ and $\Phi: \mathcal{A}^{c}(\mathcal{H}) \rightarrow \mathfrak{A}^{c}(X)$. We denote $F=\phi^{-1}(D \cap \phi(K))$. Then $F$ is $\mathcal{A}^{c}(\mathcal{H})$-extremal (see [5, Lemma 8.10]), and thus $\mathcal{H}$-extremal in $K$. Since the classes of $\mathcal{H}$-convex and $\mathcal{A}^{c}(\mathcal{H})$-convex sets coincide, $\overline{\mathrm{co}}^{\mathcal{H}}(F)=$ $\overline{\mathrm{CO}}^{\mathcal{A}^{c}(\mathcal{H})}(F)$. Let

$$
\tilde{a}(x)=a(\phi(x)), \quad x \in \overline{\mathrm{co}}^{\mathcal{H}}(F) .
$$

We claim that $\tilde{a} \in \mathcal{A}^{c}\left(\left.\mathcal{H}\right|_{\overline{\mathrm{co}}} \mathcal{H}_{(F)}\right)^{+}$. Obviously, $\tilde{a} \geq 0$. Let $x \in \overline{\mathrm{Co}} \overline{\mathcal{H}}(F)$ and $\mu \in \mathcal{M}_{x}\left(\left.\mathcal{H}\right|_{\overline{\mathrm{co}}^{\mathcal{H}}(F)}\right)$. Then $\mu \in \mathcal{M}^{1}\left(\overline{\mathrm{co}}^{\mathcal{H}}(F)\right)$, and since

$$
\overline{\mathrm{co}}^{\mathcal{H}}(F)=\overline{\mathrm{co}}^{\mathcal{A}^{c}(\mathcal{H})}(F)=\phi^{-1}(\overline{\mathrm{co}}(\phi(F)) \cap \phi(K)),
$$

the image $\phi \mu \in \mathcal{M}^{1}(X)$ under the mapping $\phi$ has support in $\overline{\mathrm{co}}(\phi(F)) \subset$ $\overline{\mathrm{co}}(D)$. Further, the measure $\mu$ considered as a measure on $K \mathcal{H}$-represents $x$. Thus $\phi \mu \mathfrak{A}^{c}(X)$-represents the point $\phi(x)$ (see [5, Proposition 4.26(c)]). Thus

$$
\mu(\tilde{a})=\mu(a \circ \phi)=\phi \mu(a)=a(\phi(x))=\tilde{a}(x) .
$$

Hence $\tilde{a}$ is an $\mathcal{A}^{c}\left(\left.\mathcal{H}\right|_{\overline{\mathrm{CO}}^{\mathcal{H}}(F)}\right)$-affine function on $\overline{\mathrm{CO}}^{\mathcal{H}}(F)$.

By (2.1), there exists a function $\tilde{b} \in \mathcal{A}^{c}(\mathcal{H})^{+}$such that $\|\tilde{b}\|_{K} \leq C\|\tilde{a}\|_{F}$ and $\tilde{b}=\tilde{a}$ on $F$. Let $b=\Phi(\tilde{b}) \in \mathfrak{A}^{c}(X)^{+}$. Then $b=a$ on $D$.

Indeed, let $s \in D$ be given. We find an $\mathfrak{A}^{c}(X)$-maximal measure $\mu \in \mathcal{M}^{1}(X)$ which $\mathfrak{A}^{c}(X)$-represents $s$ (see [5, Theorem 3.65]). Since $D$ is $\mathfrak{A}^{c}(X)$-extremal, $\mu$ is supported by $D$ and, by maximality, by $\phi(K)$ (see [5. Propositions 3.64 and 4.26(d)]). Let $\tilde{\mu} \in \mathcal{M}^{1}(K)$ satisfy $\phi \tilde{\mu}=\mu$. Then $\tilde{\mu}$ is supported by $\phi^{-1}(D \cap \phi(K))=F$. Thus

$$
\begin{aligned}
a(s) & =\mu(a)=(\phi \tilde{\mu})(a)=\int_{F} a \circ \phi \mathrm{d} \tilde{\mu}=\int_{F} \tilde{a} \mathrm{~d} \tilde{\mu}=\int_{F} \tilde{b} \mathrm{~d} \tilde{\mu} \\
& =\int_{\phi(F)} \Phi(\tilde{b}) \mathrm{d} \phi \tilde{\mu}=\mu(b)=b(s) .
\end{aligned}
$$

Obviously we have

$$
\|b\|_{X}=\|\tilde{b}\|_{K} \leq C\|\tilde{a}\|_{F} \leq C\|a\|_{D} .
$$

Thus $X=\mathbf{S}\left(\mathcal{A}^{c}(\mathcal{H})\right)$ satisfies $(H)$ in the sense of 1.1. 
In [4], the proof that simplices have the property $(H)$ is deduced from the facts that the closed convex hull of a dilated subset of a simplex is a face (see [3, p. 114]), and that affine continuous functions on a face of a simplex may be extended with preservation of norm (the proof is similar to that of [1, Theorem II.5.19]). (We recall that a closed subset $D$ of a compact convex set $X$ is said to be dilated if whenever $\mu$ is a maximal probability measure on $X$ that $\mathfrak{A}^{c}(X)$-represents a point $x \in D$ then $\mu$ is supported by $D$. Thus a closed set $D \subset X$ is dilated provided it is measure extremal. On the other hand, the set $\{0,1 / 2,1\}$ is a dilated subset of $[0,1]$ which is not measure extremal.) The following two lemmas are analogous results in the context of function spaces.

LEMMA 2.2. Let $\mathcal{H}$ be a simplicial function space and $F$ be an $\mathcal{H}$-extremal subset of $K$. Then $\overline{\mathrm{co}} \mathcal{H}(F)$ is a Choquet set.

Proof. If $F \subset K$ is a nonempty $\mathcal{H}$-extremal set, the characteristic function $\chi_{F}$ is an upper semicontinuous $\mathcal{H}$-convex function. We show that $\chi_{F}^{*}=1$ on $\overline{\mathrm{CO}}^{\mathcal{H}}(F)$.

Indeed, let $x \in \overline{\mathrm{co}}^{\mathcal{H}}(F)$ be given. By [5, Proposition 8.18], there exists a measure $\mu \in \mathcal{M}_{x}(\mathcal{H})$ supported by $F$. Then by [5, Lemma 3.21],

$$
1=\mu(F)=\mu\left(\chi_{F}\right) \leq \sup \left\{\nu\left(\chi_{F}\right): \nu \in \mathcal{M}_{x}(\mathcal{H})\right\}=\chi_{F}^{*}(x) \leq 1,
$$

which implies $\chi_{F}^{*}(x)=1$.

Now we prove that $\overline{\mathrm{co}} \mathcal{H}(F)$ is $\mathcal{H}$-extremal. To this end, let $x \in \overline{\mathrm{co}}^{\mathcal{H}}(F)$ and $\mu \in \mathcal{M}_{x}(\mathcal{H})$ be given. Let $\nu \in \mathcal{M}^{1}(K)$ be an $\mathcal{H}$-maximal measure satisfying $\mu \prec_{\mathcal{H}} \nu$ (see [5, Theorem 3.65]). Then $\nu \in \mathcal{M}_{x}(\mathcal{H})$. Since $\mathcal{H}$ is simplicial, $\chi_{F}^{*}$ is $\mathcal{H}$-affine (see [5, Theorem 6.5]). Thus by [5, Theorem 3.68],

$$
1=\chi_{F}^{*}(x)=\nu\left(\chi_{F}^{*}\right)=\nu\left(\chi_{F}\right),
$$

hence $\nu(F)=1$. By [5, Proposition 8.24],

$$
\operatorname{spt} \mu \subset \overline{\mathrm{co}}^{\mathcal{H}}(\operatorname{spt} \nu) \subset \overline{\mathrm{co}}^{\mathcal{H}}(F) \text {. }
$$

Thus $\overline{\mathrm{CO}}^{\mathcal{H}}(F)$ is an $\mathcal{H}$-extremal set and the proof is complete.

Lemma 2.3. Let $\mathcal{H}$ be simplicial, and $D$ be a closed Choquet subset of $K$. Then any $a \in \mathcal{A}^{c}\left(\left.\mathcal{H}\right|_{D}\right)^{+}$may be extended to a function in $\mathcal{A}^{c}(\mathcal{H})^{+}$with the same norm.

Proof. We define functions

$$
s(x)=\left\{\begin{array}{ll}
a(x), & x \in D, \\
0, & x \in K \backslash D,
\end{array} \quad t(x)= \begin{cases}a(x), & x \in D, \\
\|a\|, & x \in K \backslash D .\end{cases}\right.
$$

Then it is easy to verify that $s$ is $\mathcal{H}$-convex and upper semicontinuous, while $t$ is $\mathcal{H}$-concave and lower semicontinuous. We prove the desired properties 
for $s$. Concerning the $\mathcal{H}$-convexity, let $x \in K$ and $\mu \in \mathcal{M}_{x}(\mathcal{H})$. We want to show that $s(x) \leq \int_{K} f \mathrm{~d} \mu$. If $x \in D$, then spt $\mu \subseteq D$, since $D$ is a Choquet set. But $s$ coincides with $a$ on $D$, and so the desired inequality is satisfied due to the fact that $a \in \mathcal{A}^{c}(\mathcal{H})$. If $x \in K \backslash D$, then $s(x)=0$, and since $s$ is nonnegative on $K$, we are done.

Now we show that $s$ is upper semicontinuous. Let $x \in K$. If $x \in D$, then for given $\varepsilon>0$ there exists a neighborhood $U$ of $x$ such that $s=a \leq$ $a(x)+\varepsilon=s(x)+\varepsilon$ on $U \cap D$. Since on $U \backslash D$ we have $s=0<s(x)+\varepsilon$, we see that $s \leq s(x)+\varepsilon$ on $U$. On the other hand, if $x \in K \backslash D$, then since $D$ is closed, $s$ is constant on some neighborhood of $x$, and the upper semicontinuity of $s$ is proven.

Now, since $\mathcal{H}$ is simplicial, by the Edwards in-between theorem (see [5, Theorem 6.6]) there exists a function $f \in \mathcal{A}^{c}(\mathcal{H})$ such that $s \leq f \leq t$. Then $f$ is clearly a nonnegative extension of $a$ with the same norm.

We obtained the following characterization of simpliciality of a function space.

THEOREM 2.4. Let $\mathcal{H}$ be a function space on a compact Hausdorff space $K$. Then $\mathcal{H}$ is simplicial if and only if $\mathcal{H}$ has the property $(H)$ in the sense of 2.1.

Proof. It follows by Lemmas 2.2 and 2.3 that every simplicial space has the property $(H)$.

On the other hand, if $\mathcal{H}$ has the property $(H)$ in the sense of (2.1) then $\mathbf{S}\left(\mathcal{A}^{c}(\mathcal{H})\right)$ has the property $(H)$ in the sense of (1.1) by Proposition 2.1. Thus by [4, Theorem 4] the state space $\mathbf{S}\left(\mathcal{A}^{c}(\mathcal{H})\right)$ is a Choquet simplex, so $\mathcal{H}$ is simplicial.

Acknowledgements. We thank the anonymous referee for helpful suggestions leading to an improvement of the paper.

This research was supported by the research grant GAČR 17-00941S.

\section{References}

[1] E. M. Alfsen, Compact Convex Sets and Boundary Integrals, Ergeb. Math. Grenzgeb. 57, Springer, New York, 1971.

[2] T. B. Andersen, A characterization of metrizable simplexes, J. London Math. Soc. 22 (1980), 338-344.

[3] L. Asimow and A. J. Ellis, Convexity Theory and Its Applications in Functional Analysis, London Math. Soc. Monogr. 16, Academic Press, London, 1980.

[4] C. J. K. Batty, A characterisation of simplexes by an extension property, Quart. J. Math. Oxford Ser. (2) 34 (1983), 391-397.

[5] J. Lukeš, J. Malý, I. Netuka, and J. Spurný, Integral Representation Theory. Applications to Convexity, Banach Spaces and Potential Theory, de Gruyter Stud. Math. 35, de Gruyter, Berlin, 2010. 
Jakub Rondoš, Jiří Spurný

Department of Mathematical Analysis

Faculty of Mathematics and Physics

Charles University

Sokolovská 83

18675 Praha 8, Czech Republic

ORCID: 0000-0002-6201-7700 (J. Spurný)

E-mail: jakub.rondos@gmail.com

spurny@karlin.mff.cuni.cz 
